\title{
Carbon-based nanostructured materials via cluster beam deposition: a multi-technique investigation
}

\author{
A. Libassi ${ }^{\text {a }}$, P. Piseri ${ }^{\text {a }}$, P. Milani ${ }^{\text {a }}$, A. Ferrari ${ }^{\text {b }}$, C.E. Bottani ${ }^{\text {b }}$, C. Cepek ${ }^{\text {c }}$, \\ L. Giovanelli ${ }^{c}$, E. Magnano ${ }^{c}$, M. Sancrotti ${ }^{\mathrm{c}, *}$ \\ a INFM-Dipartimento di Fisica, Università di Milano, Via Celoria 16, I-20133 Milan, Italy \\ b INFM-Dipartimento di Ingegneria Nucleare, Politecnico di Milano, Piazza Leonardo da Vinci 32, I-20133 Milan, Italy \\ ${ }^{\mathrm{c}}$ Laboratorio Nazionale TASC-INFM, Padriciano 99, I-34012 Trieste, Italy
}

Received 30 July 1997; accepted for publication 3 October 1997

\begin{abstract}
The use of clusters as elemental building blocks can open new routes towards the creation of a new class of architectures and nanostructures. We report the synthesis of nanocrystalline carbon thin films produced by depositing supersonic clusters. An optimized version of a pulsed arc cluster ion source (PACIS) was utilized for the production of intense and stable ionized and neutral cluster supersonic beams. Laser photoionization and mass spectrometric techniques have been used to characterize the source, the cluster mass distribution and energy. A set of techniques was then applied to evaluate the structural and electronic properties of the thusgrown nanocrystalline carbon films encompassing Raman and C 1s X-ray photoemission spectroscopies (XPS). C 1998 Elsevier Science B.V. All rights reserved.
\end{abstract}

Keywords: Cluster beam deposition; Nanostructured materials

\section{Introduction}

In recent years, the nanoscale materials synthesis using clusters as building blocks has been a matter of speculation, and a large number of methods for cluster production and assembling have been proposed, depending on the nature of the material and on the specific application required $[1,2]$.

Here, we describe a high-efficiency PACIS recently built along with a selection of spectroscopic results relative to carbon-based nanocrystalline materials grown with it.

\footnotetext{
* Corresponding author. Fax: (+39) 40 226767; e-mail: sancrotti@sci.area.trieste.it
}

\section{Experimental}

A schematic view of the deposition apparatus is shown in Fig. 1. A clusters supersonic beam is produced by a modified version of a PACIS $[3,4]$. A carbon plasma is generated by an electrical discharge produced between two graphite rods. The plasma is mixed with a pulse of inert buffer gas to favour thermalization and cluster aggregation; the mixture is then expanded into vacuum. We have introduced several modifications compared to the original design, such as the introduction of a thermalization chamber [5], to overcome the typical PACIS instability, fast electrodes deterioration and clogging, and we have characterized the beams produced by different configurations. 


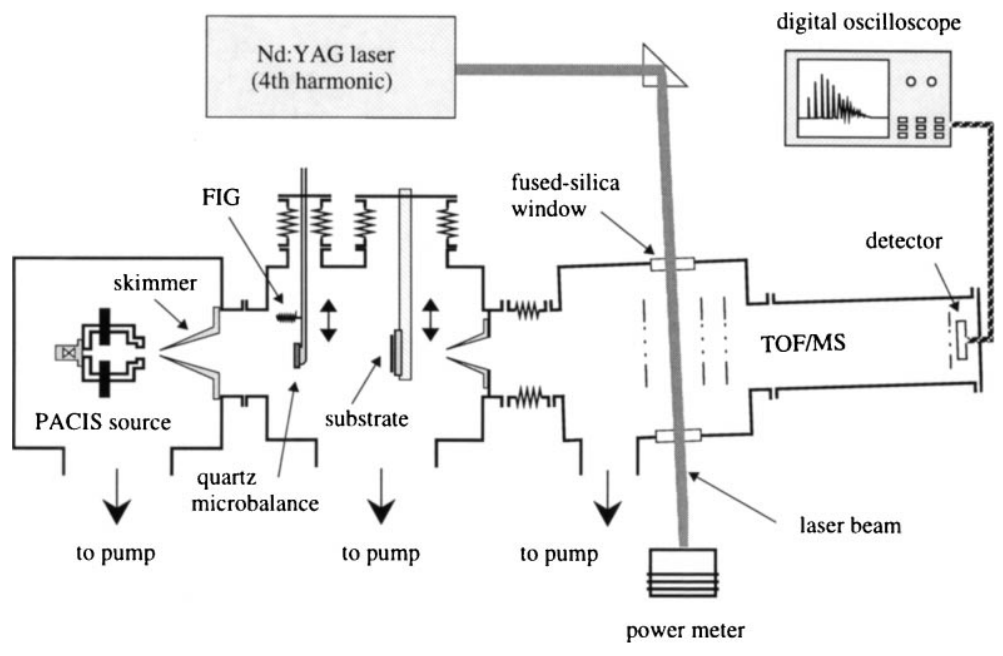

Fig. 1. Schematic view (not to scale) of the experimental apparatus used for thin film deposition.

The characterization of the different cluster mass distribution and charge states in the beam has been performed with a linear time-of-flight (TOF) mass spectrometer [6]. Neutral clusters have been ionized with the fourth harmonic $(266 \mathrm{~nm})$ of a $\mathrm{Nd}$ :YAG laser. Cluster fluxes have been characterized with a quartz microbalance and with a Faraday cup placed in the deposition chamber after the first skimmer (see Fig. 1)

Cluster-assembled specimens were grown on different substrates $[\mathrm{Si}(100)$ and poly- $\mathrm{Cu}]$ at room temperature at $\approx 1 \times 10^{-7}$ mbar.

Unpolarized Raman spectra were recorded ex-situ, at room temperature in backscattering geometry, using an I.S.A. Jobin-Yvon triple-grating spectrometer with a liquid nitrogen-cooled camera detection system. The spectral resolution was about $3 \mathrm{~cm}^{-1}$, and the 514.5-nm line of an Ar ion laser was used as excitation source; the power on the sample was about $3 \mathrm{~mW}$.

XPS has been carried out ex-situ in UHV (base pressure $\approx 1 \times 10^{-10} \mathrm{mbar}$ ), using $\mathrm{Mg} \mathrm{K}$ radiation $(h v=1253.6 \mathrm{eV})$, utilizing a double-pass cylindrical mirror analyzer for electron energy analysis. The typical overall energy resolution value was $\approx 1.2 \mathrm{eV}$. The specimens were analyzed after soft $\mathrm{Ar}^{+}$sputtering $\left(E_{\mathrm{p}}=1 \mathrm{keV}\right)$ for removing the contaminants. The surface cleanliness was checked by XPS.

\section{Results and discussion}

In Fig. 2, we show a mass spectrum of carbon neutral clusters produced with source conditions used for films deposition. The mass distribution peaks around 500 atoms per cluster, and the center of mass of the distribution is at 950 . The center of mass of the size distribution and the charge state are strongly influenced by the presence of the thermalization cavity. The clusters residence time $T_{\mathrm{R}}$ depends on parameters such as the pressure reached in the cavity, its volume and the conduc-

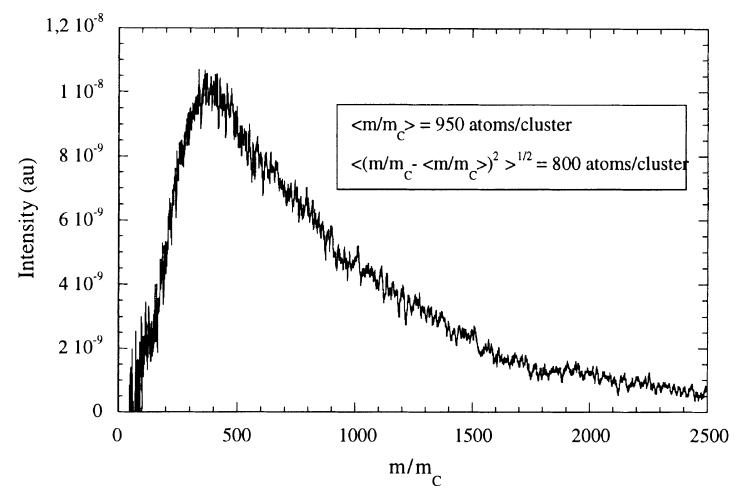

Fig. 2. Mass distribution of carbon clusters used as precursors of thin films. The TOF spectrum has been obtained by ionizing the clusters in the beam using the fourth harmonic $(266 \mathrm{~nm})$ of a Nd:Yag laser (see text). 
tance of the nozzle. In order to evaluate the real mass distribution of the deposited clusters, its evolution as a function of $T_{\mathrm{R}}$ must be taken into account. For a very large $T_{\mathrm{R}}$, clusters of several thousands of carbon atoms contributed to the mass spectra, without substantially changing the position of the center of mass of size distribution. The spectrum in Fig. 2 takes into account all the spectra produced up to $T_{\mathrm{R}}=15 \mathrm{~ms}$ after the discharge.

The ions-clusters (cations) mass distribution is different from that of neutrals: $0-1200$ atoms per cluster (mass distribution peaked at 350 atoms per clusters) for ions, $0-2000$ atoms per cluster (peaked at 500 atoms per cluster) for neutrals. This is probably due to a trade-off between cluster growth and charge neutralization: the large cluster growth requires long residence times inside the source, but this also implies a greater chance of being neutralized. Our results suggest that the assumption frequently made on the correspondence of neutral and ion cluster beam produced by the same source must be always carefully verified [2].

By growth rate measurements, performed with the quartz microbalance, the overall beam intensity is found to be in the range of $3 \times 10^{14}$ and $2 \times 10^{15} \mathrm{~s}^{-1} \mathrm{sr}^{-1}$ particles, depending on the discharge voltage. A fraction of about $10 \%$ of the total average flux is due to anions, whereas cations constitute about $2 \%$. Neutral cluster velocities are in the range of $2000 \mathrm{~m} \mathrm{~s}^{-1}$, depending on the exit time. The typical kinetic energy of a medium-size cluster is thus about $0.3 \mathrm{keV}$.

Fig. 3 shows the Raman spectrum of a film deposited at room temperature. In the low-frequency range, we observe a broad hump extending from roughly $650 \mathrm{~cm}^{-1}$ up to $800 \mathrm{~cm}^{-1}$, peaking at about $740 \mathrm{~cm}^{-1}$. The region between 1000 and $1800 \mathrm{~cm}^{-1}$ presents two peaks at $\approx 1584 \mathrm{~cm}^{-1}$ and $\approx 1363 \mathrm{~cm}^{-1}$, superimposed on a broad structure, which can be identified as the $G$ peak of crystalline graphite arising from the zone-center $\mathrm{E}_{2 \mathrm{~g}}$ mode, and the $\mathrm{D}$ peak assigned to an $\mathrm{A}_{1 \mathrm{~g}}$ zone-edge phonon activity by disorder due to the finite crystalline size $[7,8]$.

We can assume that our clusters substantially retain their individuality, due to the low-energy deposition $(<1 \mathrm{eV}$ per atom for medium size clus-

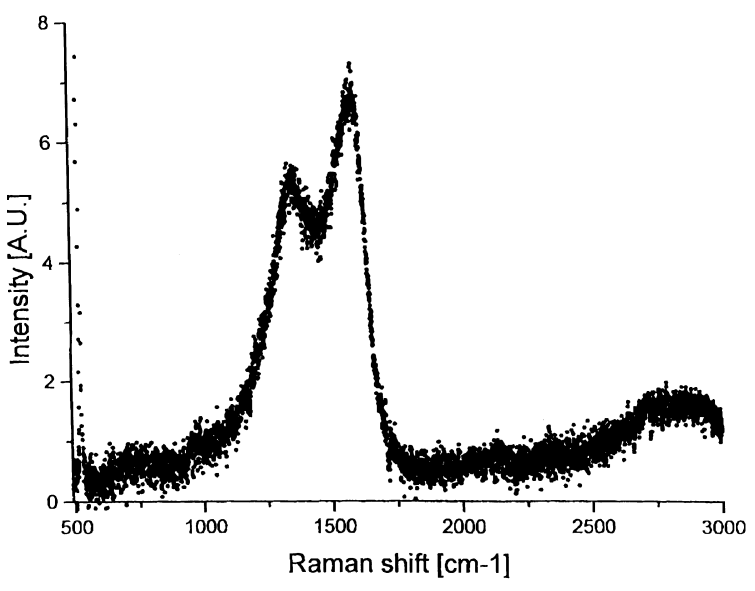

Fig. 3. Raman spectrum of a film deposited at room temperature.

ters). In our case, it is reasonable to expect a film with a $\mathrm{sp}^{2}$ character, with the presence of deformed rings and only a small number of $\mathrm{sp}^{3}$ bonds.

The predominant $\mathrm{sp}^{2}$ character of the film is confirmed by Raman spectra. The overall spectra, and in particular the intensities and the shapes of $\mathrm{G}$ and $\mathrm{D}$ bands, are typical of $\mathrm{sp}^{2}$ bonds [7,9]. The low-frequency region is also characterized by features that can be attributed to disordered $\mathrm{sp}^{2}$ bonds, as reported for different types of carbon [10]. The low-frequency region of sputtered a-C is characterized by a broad peak at $760 \mathrm{~cm}^{-1}$, which has been related to a peak in the graphite phonon density of states [11].

The feature around $1180 \mathrm{~cm}^{-1}$ deserves particular attention since it has been suggested to be related to the $\mathrm{sp}^{3}$ content in a-C films [12]. Perez and co-workers have deconvoluted the Raman spectra in the $1000-2000 \mathrm{~cm}^{-1}$ range with three Gaussians only [13], and they attributed the $1180 \mathrm{~cm}^{-1}$ peak to the presence of a microcrystalline or "amorphous" diamond phase originated by the linking of very small cage-like clusters $\mathrm{C}_{28}$ [13]. In our samples, the presence of an amorphous diamond phase is unlikely since small cage-like clusters are eventually produced and deposited together with graphitic clusters, thus preventing the formation of an $\mathrm{sp}^{3}$ network.

To confirm the predominant $\mathrm{sp}^{2}$ character and in order to compute the $\mathrm{sp}^{2}-\mathrm{sp}^{3}$ concentration in 


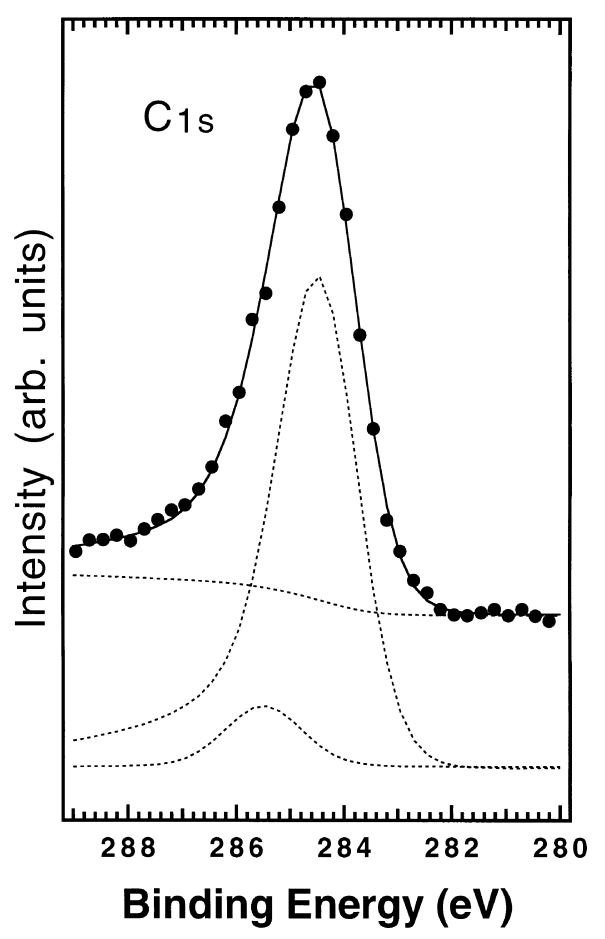

Fig. 4. XPS spectrum of a film deposited at room temperature. The filled circles represent the experimental data, the dashed lines are the components in which the spectrum is decomposed together with the integral background used in the fit, and the solid lines are the total fit.

our sample, we have tried to decompose the $\mathrm{C} 1 \mathrm{~s}$ XPS spectrum (Fig. 4) into two components, related to $\mathrm{sp}^{2}$ and $\mathrm{sp}^{3}$, respectively. A universal agreement as to what kind of data analysis protocol can positively address the quantitative evaluation of the $\mathrm{sp}^{2}-\mathrm{sp}^{3}$ ratio in a $\mathrm{C}$-based sample via core level XPS has not been established yet. Furthermore, this difficulty holds also for other spectroscopies that tend to underestimate or overestimate this ratio. As a consequence, caution has to be used, and a phenomenological approach is desired. On this basis, we have followed the procedure recently indicated in the work by J. Diaz and co-workers [14]. The C 1s spectrum has been fitted using five free parameters: the binding energy and Gaussian width of the two components and the asymmetry index $\alpha$ of the $\mathrm{sp}^{2}$ hybrid. The Lorentzian-width was fixed at $215 \mathrm{meV}$ [15]. A fit using only one component gives a value that is too large for $\alpha$ and the Gaussian width, excluding the possibility that our sample presents only an $\mathrm{sp}^{2}$ character. Our results indicate that there is $11.3 \pm 9 \%$ of $\mathrm{sp}^{3}$ hybridization, i.e. the predominant configuration in this specimen is the $\mathrm{sp}^{2}$ one. This is qualitatively in good agreement with the expectations based on Raman spectroscopy.

\section{Conclusions}

In conclusion, we have deposited and characterized nanocrystalline carbon thin films from supersonic cluster beams.

Raman and XPS spectra show that the films are essentially graphite-like with the presence of a high number of distorted $\mathrm{sp}^{2}$ bonds. The granularity of the films may have important consequences on the electronic and mechanical properties of these materials.

\section{Acknowledgements}

This work was partially financially supported by the INFM with the project CLASS.

\section{References}

[1] R.W. Siegel, Mater. Sci. Eng. B 19 (1993) 37-43.

[2] P. Melinon, V. Paillard, J. Lerme, Int. J. Mod. Phys. B 9 (1995) 339-397.

[3] H.R. Siekmann, C. Luder, J. Fachrmann, H.O. Lutz, K.H. Meiwes-Broer, Z. Phys. D 20 (1991) 417-420.

[4] P. Piseri, E. Barborini, P. Milani, Mater. Res. Soc. Symp. Proc. 400 (1996) 59-65.

[5] P. Milani, W.A. de Heer, Rev. Sci. Instrum. 61 (1990) $1835-1839$.

[6] P. Piseri, S. Iannotta, P. Milani, Int. J. Mass Spectrom. Ion Proc. 153 (1996) 23-28.

[7] R.J. Nemanich, S.A. Solin, Phys. Rev. B 20 (1979) 392.

[8] F. Tuinstra, J.L. Koenig, J. Chem. Phys. 53 (1970) 1126

[9] D.S. Knight, W.B. White, J. Mater. Res. 4 (1989) 385.

[10] F. Li, J.S. Lannin, Appl. Phys. Lett. 61 (1992) 2116.

[11] W.S. Bacsa, J.S. Lannin, D.L. Pappas, J.J. Cuomo, Phys. Rev. B 47 (1993) 10931

[12] J. Schwan, S. Ulrich, V. Batori, H. Ehrhardt, S.R.P. Silva, J. Appl. Phys. 80 (1996) 440.

[13] V. Paillard, P. Melinon, V. Dupuis, J.P. Perez, A. Perez, B. Champagnon, Phys. Rev. Lett. 71 (1993) 4170.

[14] J. Díaz, G. Paolicelli, S. Ferrer, F. Comin, Phys. Rev. B 54 (1996) 8064.

[15] C.T. Chen, F. Sette, Phys. Scr. T 31 (1990) 119. 